\title{
Correction to: Formulating Research Methods for Information Systems
}

\section{Leslie P. Willcocks, Chris Sauer, Mary C. Lacity (eds.)}

Copyrightyear is incorrectly captured in all chapters of this book. The correct copyrightyear should be 2015. This has been corrected.

The updated online version of the original chapters can be found at https://doi.org/10.1057/9781137509857, https://doi.org/10.1057/9781137509857_1, https://doi.org/10.1057/9781137509857_2, https://doi.org/10.1057/9781137509857_3, https://doi.org/10.1057/9781137509857_4, https://doi.org/10.1057/9781137509857 5, https://doi.org/10.1057/9781137509857 6, https://doi.org/10.1057/9781137509857 7 , https://doi.org/10.1057/9781137509857 8, https://doi.org/10.1057/9781137509857-9, https://doi.org/10.1057/9781137509857_10. 